\title{
Color Transparency and the Energy Evolution of Final-State Interactions in Charmonium Photoproduction*
}

\author{
S. GARDNER ${ }^{\dagger}$ \\ Continuous Electron Beam Accelerator Facility, Newport News, VA 23606
}

Introduction. To the end of understanding how color transparency may arise in certain exclusive processes ${ }^{1}$, the dynamical evolution of a photoproduced tower of $c \bar{c}$ states through a model nucleus has been calculated, for a variety of $A$ and for photon energies ranging from 8.5 to $20 \mathrm{GeV}$. That is, the final-state interactions due to multiple scattering of a produced meson with the nucleus have been calculated in a simple QCD-based quantum mechanical model.

The Model. The nucleon is modeled as a mesonic string. This is, at least, a reasonable static assumption; it has been shown in a $Y$-shaped string model for $q q q$ that the results obtained are very close to those arising from a sum of pairwise linear interactions with $\sigma_{\text {baryon }}=.54 \sigma_{\text {meson. }}{ }^{2}$ Here, then, the meson Regge string tension, $\sigma=\frac{1}{2 \pi\left(.9 \mathrm{GeV}^{-2}\right)}$, is used. The field of the string is also assumed to be uniform and purely transverse to the reaction coordinate of the $c \bar{c}$, and the "headon" field configuration seen is taken to be circular, as a crude approximation to its elliptical shape. The length of this "string" is taken to be the proton's measured charge diameter, $1.6 \mathrm{fm}$, and its width, i.e., the distance through which the $c \bar{c}$ travels, is determined to be $\tau=1.3 \pm .3 \mathrm{fm}$ from a fit in the eikonal limit to $A^{\text {eff }} / A$ of the highest energy $\left(\left\langle E_{\gamma}\right\rangle=120 \mathrm{GeV}\right)$, small $p_{T}$, incoherent photoproduction data available. ${ }^{3}$ In principle, this quantity can be measured on the lattice; a recent estimate for $q \bar{q}$ in $S U(2)_{c}$ is of the order of a fermi. The $c \bar{c}$ is modelled by a nonrelativistic harmonic oscillator, where $4 \hbar \omega=m_{\psi(4040)}-m_{\psi(3097)}$. As the confining potential is assumed to persist for all $q-\bar{q}$ separations, the picture is that of a "quenched" approximation on the lattice. The $c \bar{c}$ interaction with the nucleon string is that of a color electric dipole in $U(1)$ color, i.e., as in QED, $V_{\text {int }}=-e \vec{E} \cdot \vec{r}$; this limits consideration to exclusive channels where significant inelasticity is not present. Finally, the nucleus itself is modeled as a crystal of strings, where the spacing of the strings $\xi$ for a particular nucleus is such that $\tau+\xi=2\left(\frac{3}{4 \pi \rho_{0}}\right)^{1 / 3}$ and $\rho_{0}$ is the uniform equivalent density.

The Evolution Calculation. The computation of the development of an initial superposition of physical $c \bar{c}$ states due to interaction with the color fields of the nucleus is performed in the $c \vec{c}$ 's rest frame; one presumes this to be defined by the $J / \psi$ 's rest mass. The evolution, thus, is in time - the $c \bar{c}$ sits while the electric fields flash on and off. The time evolution is computed for a fixed set of nucleon string orientations; then, an ensemble average over all (transverse) string orientations is computed in order to yield the probabilities of measuring $J / \psi, \chi$,

- This work is supported in part by funds provided by the DOE under contract \# DEAC05-84ER40150.

1 AAUW Educational Foundation American Postdoctoral Fellow

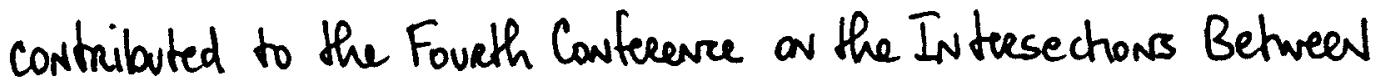

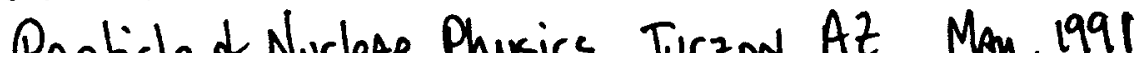


and $\psi^{\prime}$ states after traversing $N$ strings. In the $c \bar{c}$ 's rest frame, the Hamiltonian is

$$
H(\vec{r}, t)=-\frac{\hbar^{2}}{2 \mu_{c}} \nabla_{\vec{r}}^{2}+V_{H O}(\vec{r})+\sum_{i=1}^{N} V_{i n t}(\vec{r}) \Theta\left(t_{i}-t\right) \Theta\left(t-\left(t_{i}-t_{i-1}\left(1-\delta_{i 1}\right)-\xi\right)\right),
$$

where $\vec{r} \equiv \overrightarrow{r_{c}}-\overrightarrow{r_{c}}, \mu_{c}$, the reduced mass, is $.92 \mathrm{GeV}$, and $t_{i}=i(\tau+\xi)$ with $\tau[\mathrm{sec}]=\tau[\mathrm{fm}] / v_{\mathrm{c} \bar{c}}$. Writing the initial superposition of states as

$$
\langle x, y, z \mid \Psi(t=0)\rangle=\sum_{\zeta, n_{x}} C_{\zeta n_{z}}^{(i n i t)} \Phi_{n_{z}}^{h_{o}}(z) \Psi_{\zeta}(x, y),
$$

where $\zeta \equiv\left(n_{x}, n_{y}\right), \Psi_{\zeta}(x, y)=\Phi_{n_{x}}^{h o}(x) \Phi_{n_{y}}^{h o}(y)$, and with ho denoting the $c \bar{c}$ wavefunctions in free space, then at $t=t_{1}$, after passage through the first string, the coefficients $C_{\zeta n_{x}}^{(\text {init })}$ become

$$
C_{\zeta^{\prime} n_{x}^{\prime}}^{(N=1)}=\sum_{\zeta, n_{x}} \mathbf{T}_{\zeta^{\prime} n_{x}^{\prime}}^{\zeta n_{x}}\left(\theta_{1}\right) C_{\zeta n_{x}}^{(\text {init })}
$$

Here the transfer matrix $\mathbf{T}$, which may depend on the string's orientation $\theta_{1}$, is

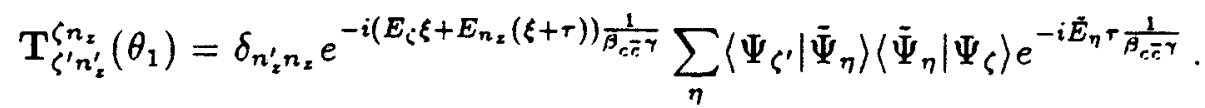

$E_{\eta}$ and $\left|\tilde{\Psi}_{\eta}\right\rangle$ are the exact two-dimensional eigenvalues - in fm $^{-1}-$ and eigenstates of the $c \bar{c}$ inside the string, and $\gamma$ is the Lorentz factor. After the $j^{\text {th }}$ string,

$$
C_{\zeta^{\prime} n_{z}^{\prime}}^{(N=j)}=\sum_{\zeta n_{z} \ldots} \mathbf{T}_{\zeta^{\prime} n_{z}^{\prime}}\left(\theta_{j}\right) \mathbf{T}\left(\theta_{j-1}\right) \ldots \mathbf{T}\left(\theta_{1}\right) C_{\zeta n_{x}}^{(\text {init })}
$$

where the additional indices, which are summed over, have been suppressed. $\theta_{1}$ defines the orientation axis, so that the final averages are of the form

$$
P_{J / \psi}^{(N=j)}=\frac{1}{(2 \pi)^{j-1}} \int_{-\pi}^{\pi} d \theta_{2} \ldots d \theta_{j}\left|C_{00}^{(N=j)}\left(0, \theta_{2}, \ldots, \theta_{j}\right)\right|^{2}
$$

The Results. In order to convert the probabilities obtained to physically meaningful observables, it is necessary (i) to make some model for the production amplitude, and (ii) to average over all possible production points in the nucleus to obtain $A^{\text {eff }} / A$. For a particular photon energy, the states which must necessarily contribute to the initial amplitude are those which can be physically produced in quasi-free kinematics with a nucleon; as the photon's coupling is point-like, it is assumed that these different states mix so as to form the smallest possible initial state. To obtain $A^{\text {eff }} / A$ for the $J / \psi$, one averages $P_{J / \psi}^{\left(N_{\text {eff }}\right)}$ over all possible production points $(z, \vec{b})$ in the nucleus - weighted by the nuclear density where $N_{\text {eff }}=\bmod \left(t(z, b) /\left(\rho_{0}(\tau+\xi)\right)\right)$, and $t(z, b)=\int_{z}^{\infty} d z^{\prime} \rho\left(z^{\prime}, b\right)$. The results are parametrized as $A^{\text {eff }} / A=A^{1+\alpha}$, where $\alpha_{\text {feed }}=-.09$ was used in the high 
energy fit for $\tau$. All calculations are in the exclusive channel where $J / \psi$ carries its maximum kinematically allowed momentum. See Table. $N_{i}$ is the number of states included in the initial amplitude, and $\alpha_{\text {feed }}$ includes the feeding of the $J / \psi$ channel from the electromagnetic decay of $\chi$ and $\psi^{\prime}$. The decrease in $\alpha$ as $E_{\gamma}$ increases is finite, though $\alpha$ changes at most by $20 \%$ over $E_{\gamma} \in[8.5,20] \mathrm{GeV}$; moreover, feeding effects tend to blur the picture. The small change in $\alpha$ over this energy range can be understood by examining the transverse size $X_{T}$ of the wave packet (this is generated dynamically by the FSI) as a function of the reaction coordinate $z$ and $E_{\gamma}$. Over some finite interval $\left(0, z_{\text {fin }}\right)$ as $E_{\gamma}$ increases, $X_{T}$ decreases, yet for still larger $z, X_{T}$ increases to values larger than it had at

\begin{tabular}{||c|c|c||}
\hline$\left(N_{i}\right) E_{r}[\mathrm{GeV}]$ & $\alpha_{\text {feed }}$ & $\alpha$ \\
\hline (1) 8.5 & $-.242 \pm .007$ & $-.269 \pm .006$ \\
(1) 12 & $-.247 \pm .006$ & $-.273 \pm .005$ \\
(1) 20 & $-.239 \pm .005$ & $-.261 \pm .008$ \\
(2) 12 & $-.238 \pm .005$ & $-.263 \pm .005$ \\
(2) 20 & $-.231 \pm .008$ & $-.249 \pm .008$ \\
(5) 20 & $-.208 \pm .006$ & $-.210 \pm .007$ \\
\hline
\end{tabular}
lower energies. Thus, the overall change in $\alpha$ is not large. $X_{T}$ is indicative of the strength of the FSI because all the individual $c \bar{c}$ nucleon interactions interfere coherently and because $V_{\text {int }}(\vec{r}) \rightarrow 0$ as $r \rightarrow 0$.

Conclusions. The $U(1)$ model used here constrains one to study the energy dependence of nearly elastic processes. Perhaps these can be isolated; however, regardless of the model's direct applicability to experiment, it is possible to make some conclusions. First, one does see a weakening of the FSI over some interval in $z$ as $E_{\gamma}$ increases, though the interactions become stronger for still larger $z$. This model, then, serves as an explicit example of how transparency (here equivalent to observation of a weakening of the FSI) may arise even when the individual interactions of the produced mesons with the medium are strong. Thus, an experimental observation of transparency does not necessarily validate the applicability of pQCD, though - if all competing backgrounds are understood - its existence makes a sub-nucleonic picture of the nuclear medium and of the particles produced in it essential. Second, the overall effect in $A^{\text {eff }} / A$ is not large for $E_{\gamma} \in[8.5,20]$ $\mathrm{GeV}$; perhaps higher energies could be required to observe transparency.

Acknowledgements. I am grateful to N. Isgur for a number of illuminating discussions and to J.W. Negele for some useful advice. I would also like to thank P. Geiger and J.D. Walecka for helpful comments.

\section{References}

1. S.J. Brodsky and A.H. Mueller, Phys. Lett. B206, 685 (1988) and references therein. See also G.R. Farrar et al., Phys. Rev. Lett. 64, 2996 (1990); B.K. Jennings and G.A. Miller, U. of Wash. preprint \# 40427-20-N90.

2. H.G. Dosch and V. Müller, Nucl. Phys. B116, 470 (1976).

3. M.D. Sokoloff et al., Phys. Rev. Lett., 57, 3003 (1986). Here the initial rms separation is $\left(m_{J / \psi}\right)^{-1}$, and the photon is assumed to have no hadronic component. However, repeating the nuclear averages assuming the photon is completely hadronic at $\left\langle E_{\gamma}\right\rangle=120 \mathrm{GeV}$ yields $\tau=1.0 \pm .2 \mathrm{fm}$.

4. A. Di Giacomo, M. Maggiore, and S. Olejník, Phys.Lett. B236, 199 (1990). 